OPEN ACCESS

Edited by:

Deqiang Duanmu,

Huazhong Agricultural University,

China

Reviewed by:

Christian Staehelin

Sun Yat-sen University, China

Huairong Pan,

Hunan University, China

*Correspondence:

Nan Yu

nyu@sibs.ac.cn

Ertao Wang

etwang@sibs.ac.cn

Specialty section:

This article was submitted to

Plant Microbe Interactions,

a section of the journal

Frontiers in Plant Science

Received: 08 December 2017 Accepted: 14 February 2018

Published: 16 March 2018

Citation:

Jin Y, Chen Z, Yang J, Mysore KS, Wen J, Huang J, Yu N and Wang E

(2018) IPD3 and IPD3L Function

Redundantly in Rhizobial and Mycorrhizal Symbioses.

Front. Plant Sci. 9:267.

doi: 10.3389/fp/s.2018.00267

\section{IPD3 and IPD3L Function Redundantly in Rhizobial and Mycorrhizal Symbioses}

\author{
Yue Jin ${ }^{1}$, Zixuan Chen ${ }^{2}$, Jun Yang ${ }^{2,3}$, Kirankumar S. Mysore ${ }^{4}$, Jiangqi Wen ${ }^{4}$, \\ Jirong Huang ${ }^{1}$, Nan $\mathrm{Yu}^{1 *}$ and Ertao Wang ${ }^{2 *}$
}

${ }^{1}$ College of Life and Environment Sciences, Shanghai Normal University, Shanghai, China, ${ }^{2}$ National Key Laboratory of Plant Molecular Genetics, CAS Center for Excellence in Molecular Plant Sciences, Institute of Plant Physiology and Ecology, Chinese Academy of Sciences, Shanghai, China, ${ }^{3}$ Collaborative Innovation Center of Crop Stress Biology, Institute of Plant Stress Biology, Henan University, Kaifeng, China, ${ }^{4}$ Plant Biology Division, Samuel Roberts Noble Foundation, Ardmore, OK, United States

Legume plants form symbiotic associations with either nitrogen-fixing bacteria or arbuscular mycorrhizal (AM) fungi, which are regulated by a set of common symbiotic signaling pathway genes. Central to the signaling pathway is the activation of the DMI3/IPD3 protein complex by $\mathrm{Ca}^{2+}$ oscillations, and the initiation of nodule organogenesis and mycorrhizal symbiosis. DMI3 is essential for rhizobial infection and nodule organogenesis; however, ipd3 mutants have been shown to be impaired only in infection thread formation but not in root nodule organogenesis in Medicago truncatula. We identified an IPD3-like (IPD3L) gene in the M. truncatula genome. A single ipd3/ mutant exhibits a normal root nodule phenotype. The ipd3//ipd3-2 double mutant is completely unable to initiate infection threads and nodule primordia. IPD3L can functionally replace IPD3 when expressed under the control of the IPD3 promoter, indicating functional redundancy between these two transcriptional regulators. We constructed a version of IPD3 that was phosphomimetic with respect to two conserved serine residues (IPD3-2D). This was sufficient to trigger root nodule organogenesis, but the increased multisite phosphorylation of IPD3 (IPD3-8D) led to low transcriptional activity, suggesting that the phosphorylation levels of IPD3 fine-tune its transcriptional activity in the root nodule symbiosis. Intriguingly, the phosphomimetic version of IPD3 triggers spontaneous root-like nodules on the roots of dmi3-1 and dmi2-1 (DM/2 is an LRR-containing receptor-like kinase gene which is required for $\mathrm{Ca}^{2+}$ spiking), but not on the roots of wild-type or ipd3/ ipd3-2 plants. In addition, fully developed arbuscules were formed in the ipd3/ ipd3-2 mutants but not the ccamk/dmi3-1 mutants. Collectively, our data indicate that, in addition to IPD3 and IPD3L, another new genetic component or other new phosphorylation sites of IPD3 function downstream of DMI3 in rhizobial and mycorrhizal symbioses.

Keywords: IPD3, IPD3L, nodule morphogenesis, phosphorylation, Medicago truncatula 


\section{INTRODUCTION}

The symbiosis between the majority of land plants and arbuscular mycorrhizal (AM) fungi results in highly branched intracellular symbiotic structures called arbuscules, which can deliver soil phosphate to the host plant (Parniske, 2008; Oldroyd, 2013; Wang et al., 2017). In turn, the plant supplies AM fungi with lipid as a carbon source (Jiang et al., 2017; Luginbuehl et al., 2017). Legume plants also form symbiosis with soil rhizobia and develop a new root-derived organ called nodule in which differentiated bacteria convert atmospheric nitrogen into a form that can be assimilated by the host plant (Oldroyd, 2013). The establishment of both root nodule and mycorrhizal symbioses is initiated through recognizing lipochitooligosaccharides, namely nodulation (Nod) factors or mycorrhizal (Myc) factors by the LysM receptor kinase proteins, and is regulated by a set of common symbiotic signaling pathway genes (Limpens et al., 2003; Madsen et al., 2003; Radutoiu et al., 2003; Arrighi et al., 2006; Zhang et al., 2007, 2015).

$\mathrm{Ca}^{2+}$ oscillations are the earliest molecular responses to the Nod or Myc factors, and the DMI3-IPD3 (named CCaMKCYCLOPS in Lotus japonicus) complex is required for the decoding of the calcium signal and initiation of nodule organogenesis in Medicago truncatula (Yano et al., 2008; Singh et al., 2014). CCaMK/DMI3 is a calcium- and calmodulindependent protein kinase (Levy et al., 2004; Mitra et al., 2004; Tirichine et al., 2006), and is composed of a CaM-binding site, a kinase domain, and 3 EF-hand motifs, and its catalytic activity is activated by either free or CaM-bound $\mathrm{Ca}^{2+}$ ions, suggesting that CCaMK/DMI3 might convert the $\mathrm{Ca}^{2+}$ signal into a protein phosphorylation read-out (Gleason et al., 2006; Tirichine et al., 2006). ccamk-13/dmi3-1 mutants are completely deficient in both the root nodule and mycorrhizal symbioses. A dmi3-1 mutant exhibits root hair swelling in response to the Nod factor, but fails to initiate infection threads or cortical cell divisions for nodule formation (Levy et al., 2004; Mitra et al., 2004). Gain of function of DMI3, by the deletion of the Cterminal regulatory domain DMI3 1-311, leads to spontaneous nodule formation in the absence of rhizobia (Gleason et al., 2006; Tirichine et al., 2006). Additionally, intraradical hyphae (Inthyphae) and arbuscules cannot be formed in the dmi3-1 mutants (Levy et al., 2004; Kistner et al., 2005). CCaMK/DMI3 acts as a central regulator in the root nodule and mycorrhizal symbioses; however, the mechanism underlying differentially activating root nodule symbiosis and arbuscular mycorrhizal symbiosis-related signal pathways by CCaMK/DMI3 is largely unknown.

IPD3/CYCLOPS encodes a nuclear-localized transcription factor with a coiled-coil motif, and is a direct phosphorylation substrate of CCaMK/DMI3 (Messinese et al., 2007; Yano et al., 2008). ipd3 mutants of M. truncatula can develop an infection pocket or short impaired infection thread, and nodule primordia during root nodule symbiosis (Yano et al., 2008; Horváth et al., 2011; Ovchinnikova et al., 2011). The Mtipd3 mutants can also form fully developed arbuscules in M. truncatula (Horváth et al., 2011). CYCLOPS is the homologous gene of IPD 3 in L. japonicus. cyclops-3, Oscyclops mutant alleles only develop Int-hypae during AM symbiosis in L. japonicus and rice, respectively (Chen et al., 2008; Yano et al., 2008; Horváth et al., 2011; Pimprikar et al., 2016). As a DNA-binding transcriptional activator, two phosphorylated serine residues (S50, S154) within the N-terminal negative regulatory domain of CYCLOPS are necessary for its activity in L. japonicus. A phosphomimetic version of CYCLOPS, in which two phosphorylated serine residues (S50, S154) are replaced by asparagic acid, can transactivate the expression of the nodulation-specific gene NODULE INCEPTION (NIN) and this is sufficient to trigger root nodule organogenesis in the absence of rhizobia (Soyano et al., 2014). However, the phosphomimetic version of CYCLOPS could not correct the plant's defective interaction with mycorrhizal fungi, indicating that other phosphorylation sites of IPD3/CYCLOPS were involved in AM symbiosis, or another unknown protein, exist downstream of DMI3 to control AM symbiosis in parallel with IPD3.

Recently, we found that IPD3 may function in a large protein complex containing other transcription factors such as DELLAs, Nodulation Signaling Pathway 2 (NSP2), and NSP1 to activate the expression of downstream genes in M. truncatula (Jin et al., 2016). DELLAs are the key negative regulators of gibberellin signaling (de Lucas et al., 2008; Feng et al., 2008) and rhizobia colonization is impaired in della mutants in M. truncatula (Fonouni-Farde et al., 2016; Jin et al., 2016). DELLAs and NSP1/NSP2 are GRAS (GAI, RGA, SCR)-type transcription factors involved in the root nodule symbiosis. Both $n s p 1$ and $n s p 2$ mutants cannot form infection pockets or initiate cortical cell division, and these mutants are therefore phenocopies of $d m i 3-$ 1 in M. truncatula (Kalo et al., 2005; Smit et al., 2005). Mtipd3 mutants can develop nodule bumps and have impaired rhizobial infection in M. truncatula (Yano et al., 2008; Jin et al., 2016). This phenotype suggests genetic redundancy downstream of DMI3.

Here, we describe the identification and characterization of an IPD3-homologous gene, IPD3-Like (IPD3L) in M. truncatula. We show that the ipd3l ipd3-2 double mutant is impaired in both rhizobial infection and root nodule organogenesis. We further show that phosphorylation levels of IPD3 fine-tune its transcriptional activity in the root nodule symbiosis. Our data also provide evidence that, in addition to IPD3/IPD3L, another new genetic component or other new phosphorylation sites of IPD3 function downstream of DMI3 to control nodule morphogenesis and arbuscule formation.

\section{MATERIALS AND METHODS}

\section{Phylogenetic Analysis}

We searched through BLAST in the phytozome database (http:// www.phytozome.net/, Goodstein et al., 2012) for sequences similar to IPD3, and the results were inspected manually. Sequences were aligned with MUSCLE, followed by manual alignment.

Phylogenetic trees were constructed using an alignment of all length protein sequences with the neighbor-joining method with the option of pairwise deletion. To test inferred phylogeny, we used bootstraps with 1,000 bootstrap replicates (Tamura et al., 2011). 


\section{Plant Materials, Bacterial and Fungal Strains and Growth Conditions}

We obtained the ipd3l mutant (NF14178) from a M. truncatula mutant population generated at the Samuel Roberts Noble Foundation (http://medicago-mutant.noble.org/mutant/ database.php). The insertion of a Tnt1 retrotransposon in mutants was confirmed by PCR (for primers see Table S1). The double mutant ipd3l ipd3-2 was generated by manual cross with ipd3-2 (NF5939) as the female parent and ipd3l (NF14178) as the male parent. The progeny of the crosses was screened through PCR using primers shown in Table S1. Phenotypic analysis was subsequently performed on the homozygous mutant and the corresponding wild-type line (R108 or Jemalong A17).

Plant seeds were scarified with $98 \%$ sulfuric acid and plated onto $1 \%$ agar medium at $4^{\circ} \mathrm{C}$ in dark. After about 3 days, they were moved to $22^{\circ} \mathrm{C}$ overnight for germination. Then the seedlings were moved to a mixed soil containing 1:1 ratio of sand and perlite. For root nodule symbiosis, plants inoculated with Sinorhizobium meliloti 1021 (Sm1021) and were grown in a greenhouse at $22^{\circ} \mathrm{C}$ with a $16 / 8 \mathrm{~h}$ light/dark cycle at $22^{\circ} \mathrm{C}$ for 4 weeks. S. meliloti 1021 was incubated in liquid Luria-Bertani medium overnight with $400 \mathrm{mg} / \mathrm{mL}$ streptomycin selection. Bacteria were pelleted at 2,000 $\mathrm{g}$ for $15 \mathrm{~min}$ and resuspended in $\mathrm{H}_{2} \mathrm{O}$ to $\mathrm{OD}_{600}=0.03$.

For AM symbiosis, plants were coincubated with Rhizophagus irregularis for 2 months. To visualize the fungal structures, roots were stained in $0.2 \mathrm{mg} / \mathrm{mL}$ WGA (wheat germ agglutinin) Alexa-fluor 488 (Javot et al., 2007). Colonization levels specified as percent root length colonized were assessed by the modified gridline intersect method (McGonigle et al., 1990). Agrobacterium rhizogenes-mediated root transformations were done according to Boisson-Dernier and associates using strain ARqual (Boisson-Dernier et al., 2001).

\section{DNA Constructs}

For yeast two-hybrid constructs, the appropriate genes were cloned into the Gateway donor vector pEntry-topo-SD (Table S1), sequenced and then recombined into the pGBKT7GW and pGADT7GW vectors by LR reactions (Invitrogen). For subcellular localization vectors, IPD3 and IPD $3 \mathrm{~L}$ were cloned into pSAT4-eGFP-N1 with appropriate restriction enzyme cleavage sites (Table S1). For protein expression in Escherichia coli, pET28a, and pMal-C2X were used (for primers see Table S1). For transient expression assays, the promoter of ERN1 was fused to the pCAMBIA2300 vector. For transgenic hairy root vectors, 3XHA-IPD3, and its point mutations (S14, S43, S50, T59, S81, S88, S155, and S407) were amplified and inserted into the Gateway donor vector pEntry-topo-SD and then recombined into the pK7GW2-R vectors by LR reactions. For GUS staining assay, the inferred IPD3 promoter (1048 bp upstream of the start codon according to Ovchinnikova et al., 2011), and IPD3L promoter (3,000 bp upstream of the start codon) were cloned to the vector pEntry-topo-SD, and then recombined into pBGWFS7. For complementary experiments, full-length IPD3L CDS was amplified from cDNA, cloned to the vector pEntrytopo-SD, and then recombined into pK7WG2-R of which the p35S promoter was replaced by IPD3 promoter with appropriate restriction enzyme cleavage sites. All primers are listed in Table S1.

\section{Protein Subcellular Localization and Yeast Two-Hybrid Analysis}

Plasmids were extracted according to the manual of NucleoBond Xtra Midi Plus (Macherey-Nagel). Isolation of Arabidopsis mesophyll protoplasts and transient gene expression were performed as previously described (Yoo et al., 2007). Enhanced green fluorescent protein (eGFP) fluorescence was recorded by a confocal laser scanning microscope (FluoView FV1000; Olympus).

Yeast two-hybrid analysis was carried out as described in the yeast handbook PT3024-1 (Clontech). The transformed AH109 yeast strains were selected on synthetic dropout (SD) plates lacking Leu and Trp (-LW). The expression of HIS3 and ADE2 reporter genes was assessed by colony growth of yeast strains transformed with DMI3, IPD3 or IPD3L genes on SD-LWHA (-Leu, -Trp, -His, and -Ade) plates.

\section{GUS Staining and Enzymatic Assays}

Transgenic plants hairy roots which were mediated by Agrobacterium rhizogenes, containing promoter-GUS constructs were used for the GUS staining assay. The roots were putted into the staining buffer and a vacuum was applied for $10 \mathrm{~min}$. Roots were then incubated for $12 \mathrm{~h}$ or $24 \mathrm{~h}$ at $37^{\circ} \mathrm{C}$. For enzymatic GUS assays, leaf tissues were ground in liquid nitrogen and homogenized in the GUS extraction buffer (50 mM sodium phosphate, $\mathrm{pH} 7.5,10 \mathrm{mM} \beta$-mercaptoethanol, $10 \mathrm{mM} \mathrm{Na} 2$ EDTA, $0.1 \%$ Triton $\mathrm{X}-100$, and $0.1 \%$ sodium laurylsarcosine) for total protein extraction. GUS activities were measured fluorimetrically using $1 \mathrm{mg}$ of total protein extract as described previously (Boisson-Dernier et al., 2005).

\section{In Vitro Phosphorylation and Mass Spectrometric Analysis}

IPD3 was expressed from pET28a in E. coli strain Rosetta (TransGen Biotech). Expression products were affinity purified via nickel-agarose (Qiagen) under denaturing conditions using $8 \mathrm{M}$ urea. Denatured proteins were refolded by stepwise dialysis (Yano et al., 2008). Purification of MBP-DMI3, HIS-IPD3, and HIS-IPD3L was performed according to the protocols of E8200 (New England Biolab) and of BioSprint96 (Qiagen). IPD3 was phosphorylated in vitro by DMI3 as described (Jin et al., 2016). Each reaction was carried out with $1 \mathrm{mg}$ MBP-DMI3 protein, $0.2 \mathrm{mM} \mathrm{CaCl}_{2}, 0.5 \mathrm{mM}$ bovine CaM, $2 \mathrm{mg}$ 6XHIS-IPD3 as substrate. Mass spectrometric analysis, in-gel digestion of phosphorylated IPD3, liquid chromatography (LC)-MS data acquisition and database searches were performed by Shanghai Applied Protein Technology Co. Ltd (http://www.aptbiotech. com).

\section{Gene Expression Analysis}

Total RNA was extracted from $M$. truncatula hairy roots 4 weeks post transformation using the TransZol UP reagent (TransGen). RNA samples were treated with Ambion DNA Removal Kit and absence of DNA contamination was confirmed by PCR. First strand cDNA was synthesized with oligo (dT) 18 from 1 mg RNA 
with M-MLV reverse transcriptase (Takara). Real-time RT-PCR analysis was carried out in a CFX96 Real-Time PCR machine (BioRad) using $1 \mu \mathrm{l}$ of diluted (1:20) cDNA in a total reaction volume of $10 \mu \mathrm{l}$ containing SYBR Green Master Mix (Takara) and primers. Thermal cycling conditions were: $95^{\circ} \mathrm{C} 1 \mathrm{~min}, 45$ cycles of $95^{\circ} \mathrm{C} 10 \mathrm{~s}, 60^{\circ} \mathrm{C} 30 \mathrm{~s}$, followed by dissociation curve analysis. Relative expression was normalized to the reference gene ubiquitin. Mean and standard error values were calculated from three biological replicates. More than ten plants were used for RNA extraction.

\section{Transient Expression in Nicotiana benthamiana Leaves}

Agrobacterium tumefaciens GV3101 strains containing binary vectors harboring either 3XHA-tagged IPD3 or its mutations and proERN1:GUS fusion were grown on LB medium supplemented with the appropriate antibiotics at $28^{\circ} \mathrm{C}$, harvested and resuspended in suspension buffer $\left(10 \mathrm{mM} \mathrm{MgCl}_{2}, 10 \mathrm{mM}\right.$ MES, $200 \mu \mathrm{M}$ acetosyringone, $\mathrm{pH}=7.0$ ) before infiltration of Nicotiana benthamiana leaves. Leaf discs were harvested $36 \mathrm{~h}$ post-infiltration and frozen in liquid nitrogen prior to quantitative enzymatic GUS fluorimetric assays. The expressed proteins in Nicotiana benthamiana leaves were detected by western blot analysis using anti-HA (Sigma) antibody (the uncropped versions of blots in Figure S7).

\section{RESULTS}

\section{Identification and Cloning of an IPD3-Like Gene}

In ipd3-2 mutants, the development of infection threads is aborted and nodule development is prematurely arrested (Yano et al., 2008; Horváth et al., 2011; Ovchinnikova et al., 2011). The phenotype of ipd3-2 is clearly different from dmi3-1 mutants, in which both infection threads and nodule initiation are aborted. Therefore, we speculated that an IPD3 homologous gene might function redundantly with IPD3 in the root nodule symbiosis. We searched for a homologous protein sequence in the phytozome database (https://phytozome.jgi.doe.gov/pz/ portal.html) using the IPD3 full-length protein sequence, and a protein composed of 626 AA was found, which we named IPD3like (IPD3L). We employed reverse-transcription polymerase chain reaction (RT-PCR) to amplify IPD $3 \mathrm{~L} \mathrm{cDNA}$, and found that there were 9 more nucleotides at the 256 site in the amplified fragment than predicted from the genome sequence (http://www. medicagogenome.org) (Data set S1). IPD3L has a longer Cterminus than IPD3 (Figure S2). A phylogenetic tree showed that IPD3L is more closely related to IPD3 than to an IPD3 homolog in the non-mycorrhizal plant Arabidopsis, suggesting that IPD3L might function in root nodule and mycorrhizal symbioses (Figure $\mathrm{S} 1$ ).

\section{The ipd3I ipd3-2 Double Mutant Is Impaired in Both Rhizobial Infection and Root Nodule Development}

IPD3 and DMI3 both localize to the nucleus and decode the calcium oscillation in the nucleus (Messinese et al., 2007; Yano et al., 2008). To test whether IPD3L also was localized in the nucleus, we constructed a vector expressing IPD3L-eGFP driven by a $35 \mathrm{~S}$ promoter. Arabidopsis protoplasts expressing the control p35S:eGFP vector exhibited strong fluorescence in both cytoplasmic and nuclear compartments (Figure S3A). Fusion of eGFP to the full-length IPD3 and IPD3L resulted in specific accumulation of GFP signals in the nucleus (Figure S3A). Meanwhile, we found that IPD3L can interact with DMI3 in a yeast two-hybrid assay (Figure S3B). These results suggested that IPD3L might function redundantly with IPD3 in the root nodule and mycorrhizal symbioses.

To further explore the function of $I P D 3 L$, we ordered ipd $3 l$ mutants containing Tnt1 insertions (NF14178) from a mutant population generated at the Samuel Roberts Noble Foundation (http://medicago-mutant.noble.org/mutant/database.php).

Transcripts of IPD3L was absent in the ipd $3 l$ mutant line (Figures S2, S3). The rhizobial infection and nodule development in the ipd3l mutant were comparable to wild-type (R108) (Figure 1A). Considering the possibility of functional redundancy, we generated an ipd3l ipd3-2 double mutant by crossing the ipd3-2 (NF5939) with ipd3l (NF14178) mutant lines. Double mutants were obtained from the progeny through PCR analysis for further phenotypic analysis (Table S1). Intriguingly, no nodule primordia were formed in the ipd3l ipd3-2 double mutants (Figures 1A,B), which is a phenocopy of the dmi3-1 mutant. Further investigation showed that only infection pockets were formed on the roots of the ipd3l ipd3-2 mutant (Figures 1C,D), indicating that IPD3L and IPD3 function redundantly in the root nodule symbiosis.

\section{The Expression of IPD3L in Root Nodule Symbiosis}

To further investigate the role of $I P D 3 L$ in the root nodule symbiosis, we compared the expression profiles of IPD3 (pIPD3:GUS) and IPD3L (pIPD3L:GUS). IPD3 and IPD3L expression in roots was detected in the absence of rhizobial inoculation (Figure 2A). In the rhizobia-inoculated roots, IPD3 expression was detected strongly through the whole nodule, while pIPD3L:GUS expression was slightly detected in the meristem region and vascular tissues at 2 weeks post-inoculation (wpi) (Figure 2A). At 4 wpi, the GUS activity of IPD3 was detected most strongly in the region from the nodule meristem region to the nitrogen-fixation zone, and the expression pattern of IPD3L was very similar to that of $I P D 3$, but $I P D 3 L$ was expressed at much lower levels at 2 and 4 wpi (Figure 2A). To verify the difference in the expression levels, real-time-PCR was further employed to reveal the expression levels of IPD3 and IPD3L. IPD3 expression levels were approximately 100 times higher than that of $I P D 3 L$ in roots with or without rhizobial inoculation (Figures 2B,C). Thus, our results showed that IPD3L functions redundantly with $I P D 3$, although the expression level of $I P D 3 L$ is much lower than IPD3 in roots.

\section{IPD3L Can Functionally Replace IPD3 When Expressed Under the Control of the IPD3 Promoter}

Only short infection threads and bumps developed on the ipd3-2 mutant roots, whereas the normal pink nodules were observed 

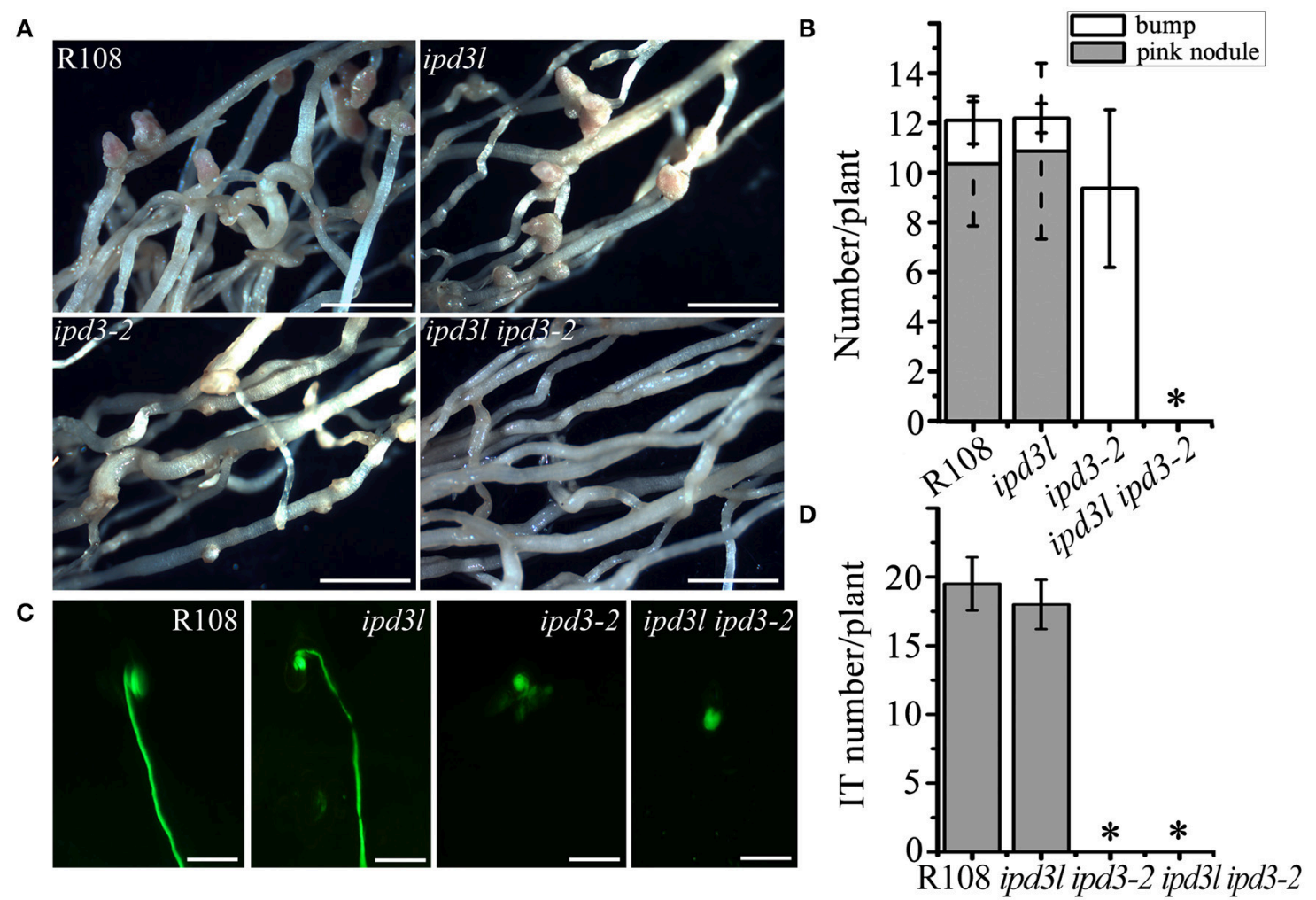

FIGURE 1 | ipd3/ ipd3-2 mutant is impaired in nodule development. (A) Phenotypic analyses of wild-type R108, ipd3/, ipd3-2, and ipd3/ ipd3-2 double mutants at 4 weeks post-inoculation (wpi) with Sinorhizobium meliloti (strain 1021). Pink nodules were developed on R108 and ipd3/ roots. Uninfected nodules (bump) were found on ipd3-2 roots. No pink nodules or bumps were observed on the ipd3/ ipd3-2 double mutant roots. (B) Quantitative analysis of nodules grown on R108, ipd3/, ipd3-2, and ipd3/ ipd3-2 roots. (C) Representative images of infection threads in the wild-type, ipd3/, ipd3-2 and ipd3/ ipd3-2 mutants. S. meliloti 1021 is labeled by green fluorescent protein (GFP). (D) Quantitative analysis of infection threads (IT) grown on R108, ipd3/, ipd3-2, and ipd3/ ipd3-2 roots. Scale bars correspond to $1 \mathrm{~mm}$ in $\mathbf{( A , B )} 100 \mu \mathrm{m}$ in (C). These experiments were repeated three times with similar results. The error bars indicate standard error. The asterisk indicates a significant decrease relative to the control with Student's $t$-test $\left({ }^{\star} P \leq 0.01\right)$.

on the ipd3l mutant roots (Figure 1). This implies that IPD $3 L$ and IPD3 also function differently in the root nodule symbiosis. To address the question of whether IPD3L can functionally replace IPD3, cross-complementation studies were performed by transforming an ipd3-2 mutant line with IPD $3 L$ driven by the IPD3 promoter. Fully developed and infected nodules were formed on ipd3-2 hairy roots transformed with IPD3L, but not on ipd3-2 hairy roots transformed with the empty vector (Figure 3). Thus, when expressed under the control of the IPD3 promoter, $I P D 3 L$ can functionally replace $I P D 3$ in the root nodule symbiosis.

\section{Serine Residue Phosphorylation of IPD3 Fine-Tunes Root Nodule Symbiosis}

The availability of the non-nodulating double mutant ipd $3 l$ ipd3-2 opened the possibility to characterize different phosphoablative/-mimetic IPD3 variants. No pink nodules were observed on ccamk-3 roots transformed with CYCLOPSS50D-S154D in L. japonicus (Singh et al., 2014). To identify other amino acids of IPD3 phosphorylated by DMI3 functioned in the root nodule symbiosis, we purified $6 \times$ HIS-IPD3 protein and MBP-DMI3 protein from E. coli and performed
DMI3-mediated IPD3 phosphorylation experiments in vitro. Three phosphorylated serine residues (S14, S81, S88) and one phosphorylated threonine residue (T59) were detected by mass spectrometry (Figure S5A). Among these sites, only one residue (S14) had a corresponding phosphorylation site in CYCLOPS (Singh et al., 2014). This discrepancy may be due to the different $\mathrm{Ca}^{2+}$ concentrations used in the studies. All together, eight potential phosphorylated residues of IPD3 protein (S14, S43, S50, T59, S81, S88, S155, and S407), including the S43 reported by Grimsrud et al. (2010), have been found so far from alignment between IPD3 and CYCLOPS (Figure S5B). This data suggest that DMI3 could phosphorylate additional residues in IPD3-2D and that these sites could be important for full IPD3 function.

To examine whether phosphorylation of the newly identified sites is essential for the root nodule symbiosis, we generated single phosphoablative mutant versions of IPD3 and analyzed the restoration of the root nodule symbiosis in transformed ipd $3 l$ ipd3-2 mutants with these phosphoablative mutant versions. In these variants, T59, S81, and S88 were individually substituted by alanine (A) (Table S2). The number and morphology of pink nodules on the hairy roots of plants containing alanine replacements at the three sites was the same as the control. 

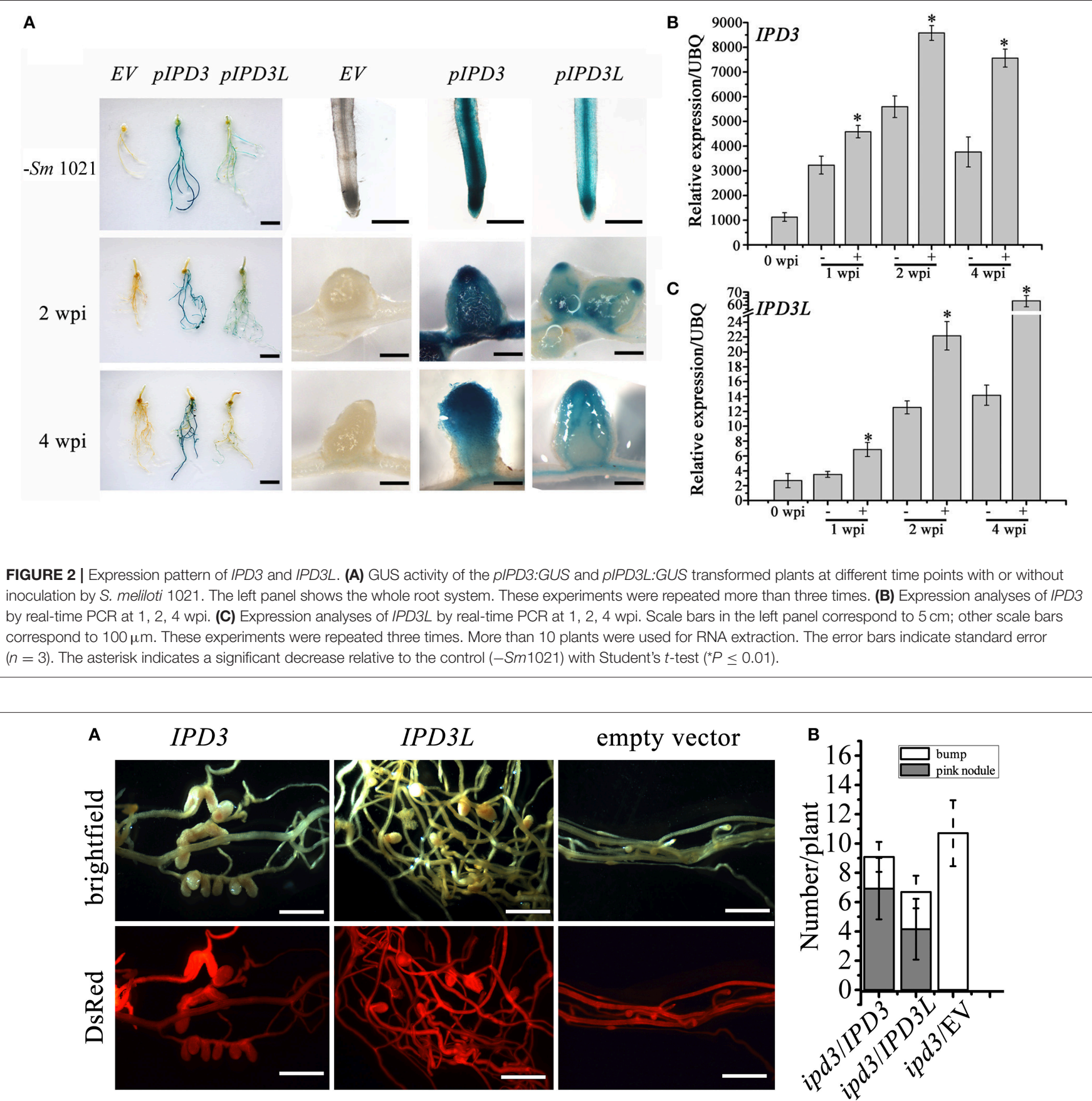

FIGURE 3 | IPD3L rescues the phenotype of ipd3-2. (A) The nodulation phenotypes of ipd3-2 mutant roots transformed with IPD3, or IPD3L or the empty vector control (expressed under the control of the $1.1 \mathrm{~kb}$ IPD3 promoter) at 4 wpi. Transformed roots were screened with DsRed reporter (contained in the binary vector). (B) Quantitative analysis of nodule number on ipd3-2 mutant roots transformed with IPD3, or IPD3L or the empty vector control at 4 wpi. Scale bars correspond to $1 \mathrm{~mm}$. These experiments were repeated three times with similar results.

Therefore, we further generated multisite phosphoablative/mimetic mutant versions of S14, S43, S50, T59, S81, S88, S155, and $\mathrm{S} 407$ to test their function in nodule formation. Replacing serine/threonine with aspartate (D) can result in a gain-offunction activity of the protein (phosphomimetic version of the protein). We found that the IPD3-2D version (S50, S155) could rescue nodule formation on the ipd3l ipd3-2 double mutant (Table S3). Strikingly, IPD3-8D decreased the nodule number on the ipd3l ipd3-2 mutant even though the morphology of pink nodules is not different from that on the wild-type plants. To test whether this phenotype was caused by some instability of the protein, we performed a western blot with an anti-HA-HRP 
antibody. We found that 3xHA-IPD3-WT and 3xHA-IPD3-8D were expressed at similar levels in ipd3l ipd3-2 mutant roots (Figure S6A).

Consistent with the decreased nodule number phenotype, the expression level of nodule specific genes, namely NIN, ERN1,
Enod11 and FLOT4 were lower in the IPD3-8D transformed hairy roots than that in IPD3-WT transgenic roots (Figure 4), but higher than that in the empty vector, IPD3-2A, and IPD3$8 A$ roots. Meanwhile, we examined the transcriptional activity of different phosphomimetic versions of IPD3 in N. benthamiana

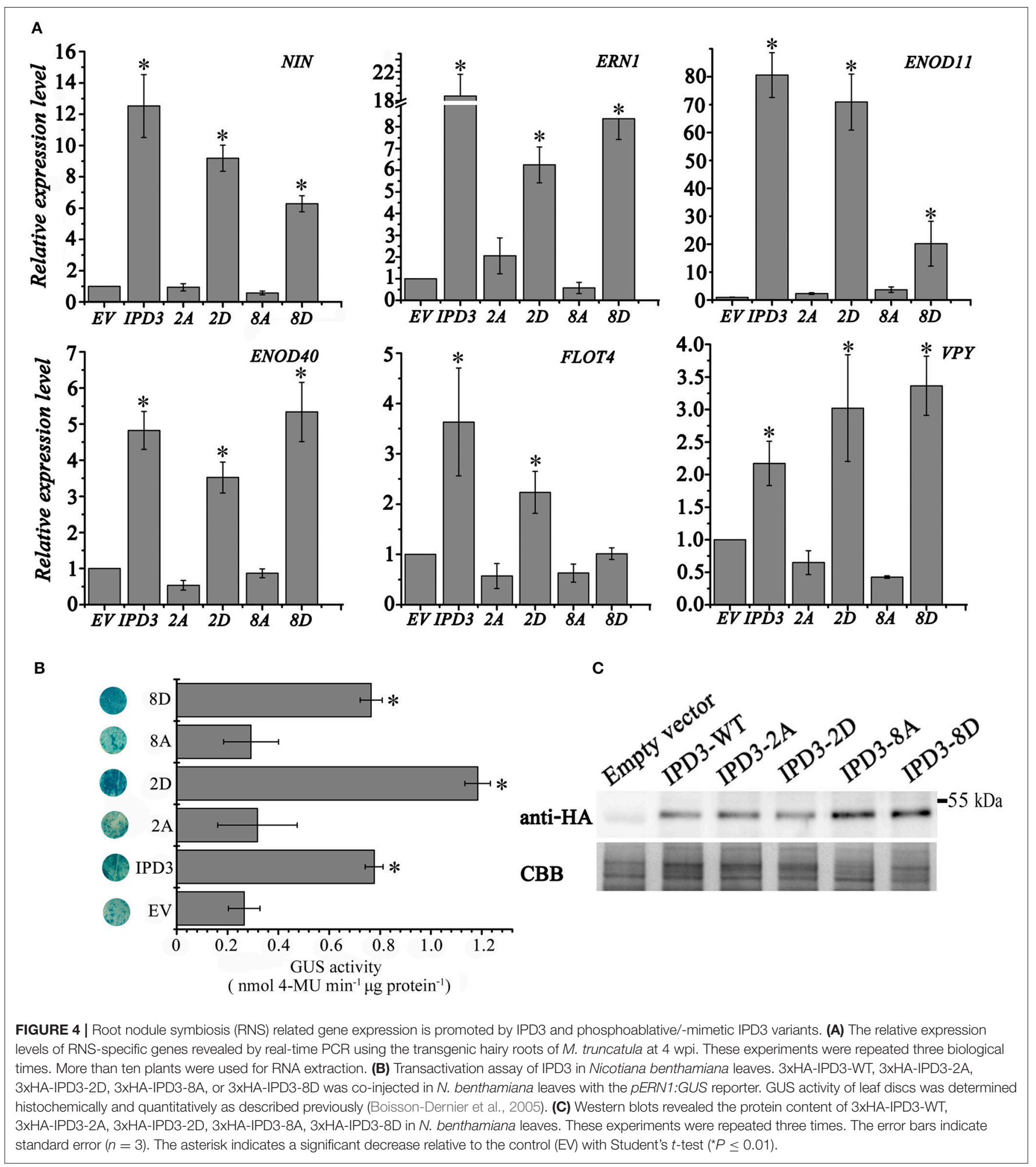


leaves. Interestingly, empty vector, IPD3-2A, and IPD3-8A could cause a faint reporter gene expression, while IPD3 and IPD3-8D were sufficient to induce a significant expression of ERN1. IPD32D could further active expression of ERN1 in N. benthamiana leaves (Figure 4; Figure S7). Together, our data suggest that IPD3-2D plays a positive role, while IPD3-8D plays a negative role in the root nodule symbiosis. However, the detail of the mechanism needs to be further explored.

CYCLOPS has been identified as a phosphorylation substrate of CCaMK and only uninfected nodules were observed on ccamk3 roots transformed with CYCLOPS-S50D-S154D in L. japonicus (Singh et al., 2014). Consistent with this, no pink nodules were found on dmi3-1 roots transformed with IPD3-2D and IPD3$8 \mathrm{D}$ at 4 wpi in $M$. truncatula (Table S4), indicating that this multisite phosphorylation of IPD3 (IPD3-2D/8D) by DMI3 is not sufficient to trigger the downstream signaling for rhizobial infection.

\section{IPD3-2D Induces Spontaneous Root Nodules}

Gain of function versions of CCaMK/DMI3 are sufficient to trigger spontaneous nodule organogenesis in the absence of rhizobia (Gleason et al., 2006; Tirichine et al., 2006). We found that IPD3-2D was also sufficient to trigger spontaneous nodules on the wild-type and ipd3l ipd3-2 roots in the absence of rhizobia (Figure 5, Table S5), which is consistent with the data reported in L. japonicus (Singh et al., 2014). Strikingly, the spontaneous nodules on dmi3-1 and dmi2-1 roots triggered by IPD3-2D were root-like nodules with many similarities to those on Mtnoot (nodule root in M. truncatula) and Pscoch (cochleata in Pisum sativum) roots, which are characterized by the abnormal development of roots from the nodule (Couzigou et al., 2012) (Figure 5). This observation indicated that a signal was activated by CCaMK/DMI3 to maintain the normal spontaneous development of nodules triggered by
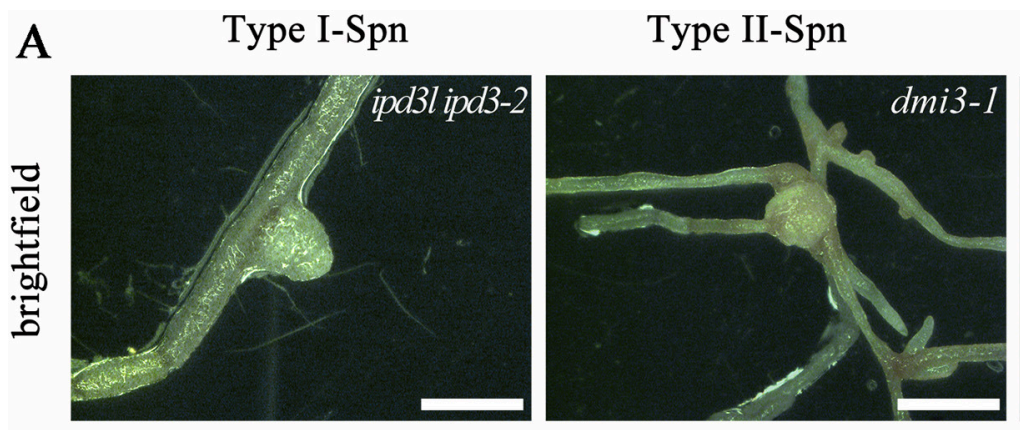

\section{Type III-Spn}
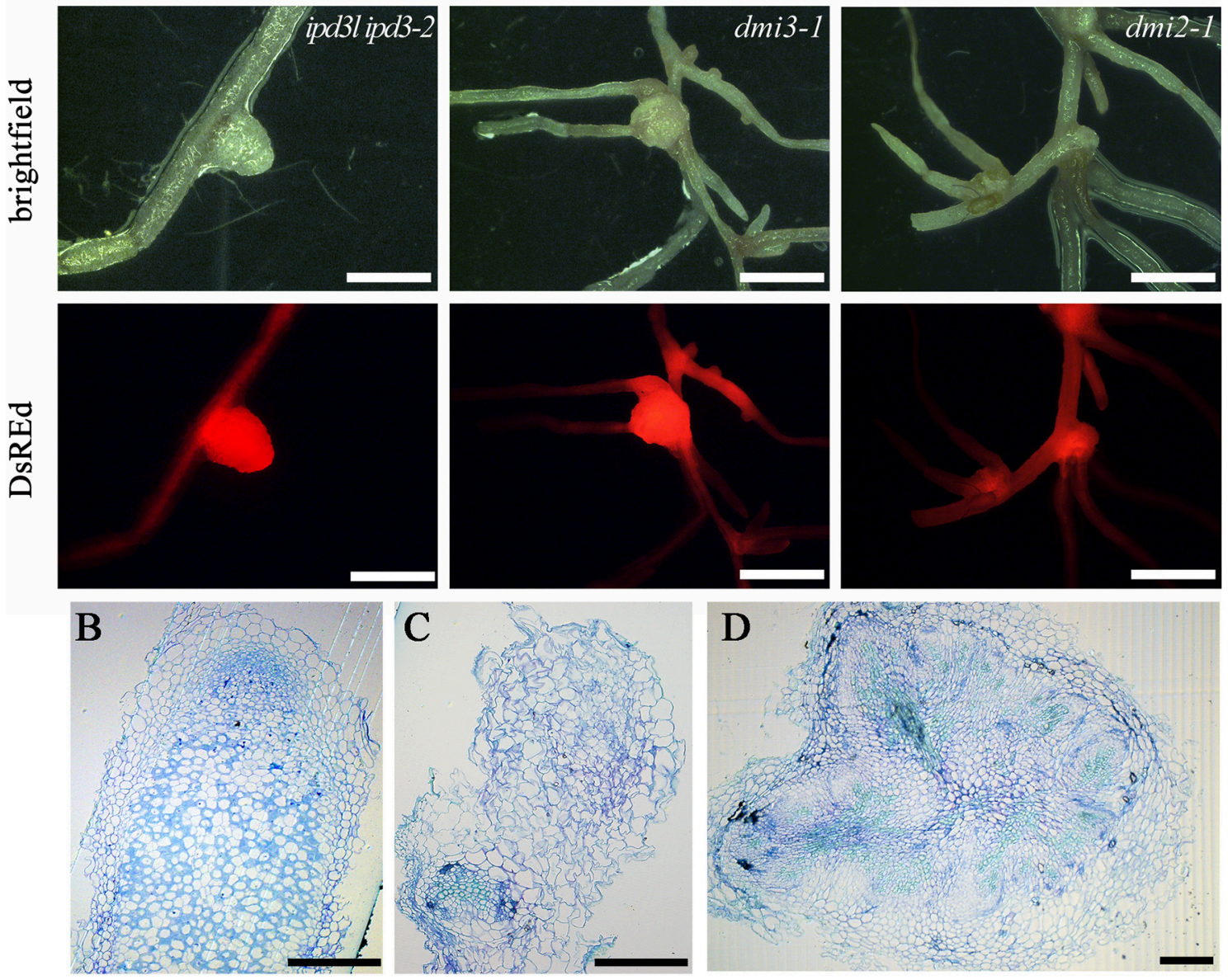

FIGURE 5 | IPD3-2D triggers spontaneous root-like nodules. (A) Type I spontaneous nodules developed on the ipd3/ ipd3-2 hairy roots transformed with the phosphomimetic IPD3-2D mutant version, Type II and III developed on the hairy roots of dmi3-1 and dmi2-1 transformed with the phosphomimetic IPD3-2D mutant version. Spontaneous nodules were observed and scored at 8 weeks transformed into the soil. (B) Toluidine blue-stained section of a pink nodule induced by $S$. meliloti 1021. (C) Toluidine blue-stained sections of type I spontaneous root-like nodules. (D) Toluidine blue-stained sections of type II spontaneous root-like nodules. These experiments were repeated three times with similar results. Scale bars in (A) correspond to $1 \mathrm{~mm}$; scale bars in (B-D) correspond to $0.1 \mathrm{~mm}$. 
IPD3-2D. This signal may be a new protein or some new phosphoralation sites of IPD3. In addition, the number of nodules on the hairy roots transformed with IPD3-8D vectors was significantly decreased compared with roots transformed with IPD3-2D in ipd3l ipd3-2, dmi3-1, and dmi2-1 mutants (Table S5).

Since IPD3-2D induced spontaneous root nodules on dmi31 mutants, we asked whether formation of spontanious nodules induced by an autoactive form of DMI3 (DMI3 1-311) is dependent on IPD3. We introduced the DMI3 1-311 into the ipd3l ipd3-2 mutant via hairy root transformation. Only several surface protuberances (3/25) were found (Figure S6), but no spontaneous nodules were formed on ipd3l ipd3-2 mutants, indicating that $I P D 3 / I P D 3 L$ is required for spontaneous nodules induced by expression of a deregulated form of DMI3.

\section{Fully Developed Arbuscules Are Formed in ipd3l ipd3-2 Mutants}

It has been shown that IPD3 plays an important role in mycorrhizal symbiosis (Yano et al., 2008; Horváth et al., 2011; Pimprikar et al., 2016). Therefore, we assessed whether IPD3L also functions redundantly in mycorrhizal symbiosis. Wild-type, $i p d 3-2$, ipd3l, and ipd3l ipd3-2 roots inoculated with sands mixed with Rhizophagus irregularis. Surprisingly, fully developed arbuscules were found in the ipd3l ipd3-2 roots (Figure 6A), although the frequency of fungal colonization was significantly reduced in the ipd3l ipd3-2 mutant compared with wild-type plants (Figures 6B,C). In dmi3-1 mutants, mycorrhizal hyphae were observed only on the root surface. Interestingly, our results differ from previous observations of ipd3-2 roots (Horváth et al., 2011), where fully developed arbuscules were not observed in the mutant roots inoculated with $R$. intraradices. To verify our results, we repeated this experiment four times under our conditions, and highly branching arbuscules were consistently observed in roots of both the ipd3-2 and the ipd3l ipd3-2 double mutant (Figure 6). Consistent with reduced AM colonization, the expression levels of AM marker genes, PT4 (PHOSPHATE TRANSPORTER 4) and HA-1( $H^{+}$-ATPase) (Javot et al., 2007; Wang et al., 2014), were seriously decreased in the ipd3l ipd32 plants. Taken together, our data indicate that another genetic component, in addition to IPD3/IPD3L, functions downstream of DMI3 to regulate arbuscule development in mycorrhizal roots.

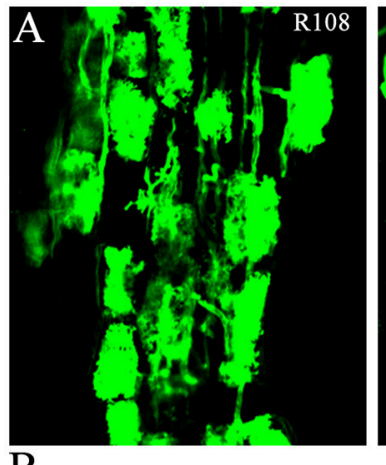

B

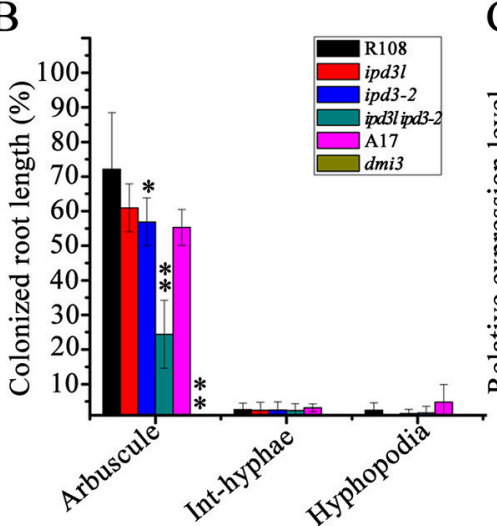

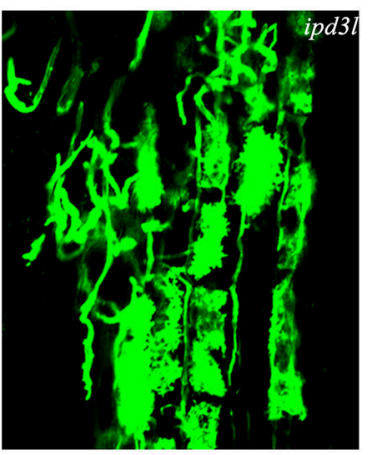

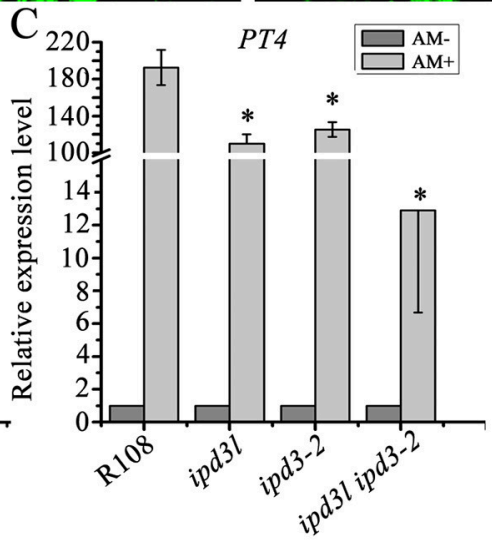

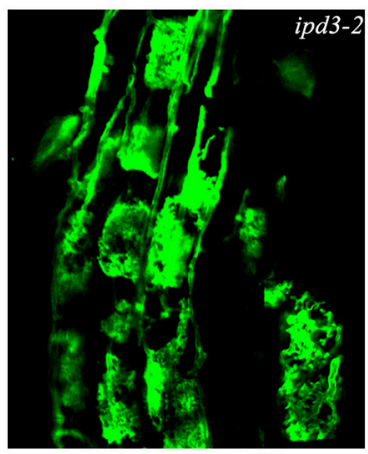

D

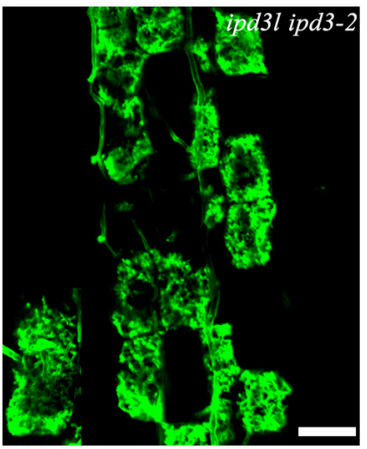

$H A-1$

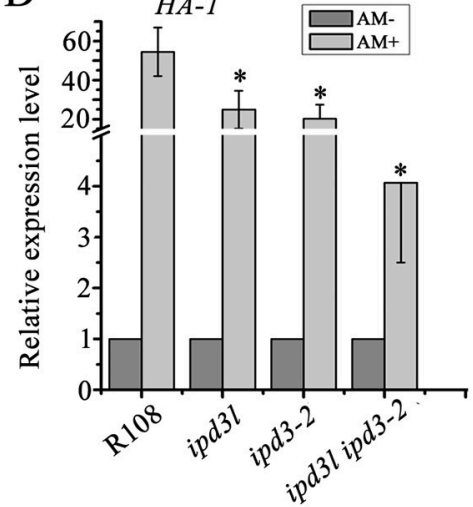

FIGURE 6 | Fully developed arbuscules are formed in the ipd3/ ipd3-2 mutant roots. (A) Laser scanning confocal images of wild-type, ipd3/, ipd3-2, and ipd3/ ipd3-2 mutants at 8 wpi. The fungus was stained with $0.2 \mathrm{mg} / \mathrm{mL}$ WGA-Alexa-Fluor 488 . Scale bars correspond to $20 \mu \mathrm{m}$. (B) Percent root length colonization of wild-type, ipd3-2, ipd3/, and ipd3/ ipd3-2 roots by Rhizophagus irregularis at 45 days post-inoculation (dpi). The degree of root colonization was determined by gridline intersect method. These experiments were repeated four times with similar results. The asterisk indicates a significant decrease compared to the control with Student's $t$-test $\left({ }^{\star} P \leq 0.05 ;{ }^{*} P \leq 0.01\right)$. (C,D) Expression analyses of arbuscular mycorrhizal specific marker genes by real-time PCR. These experiments were repeated three biological times. More than ten plants were used for RNA extraction. The error bars indicate standard error $(n=3)$. The asterisk indicates a significant decrease compared to the control with Student's $t$-test ( $\left.{ }^{*} P \leq 0.01\right)$. 


\section{DISCUSSION}

The establishment of nitrogen-fixing nodules in legumes involves in the decoder of calcium spiking and regulation network of transcription factors. Forward and reverse genetic approaches have demonstrated that these transcription factors play central roles in root nodule symbiosis. However, despite clear-cut infection thread defect phenotypes, many transcription factors mutants, such as ern1, ipd3, nf-ya1, are still able to partially initiate early symbiotic signaling to form nodule primordia (Middleton et al., 2007; Yano et al., 2008; Horváth et al., 2011; Ovchinnikova et al., 2011; Cerri et al., 2012, 2016; Laloum et al., 2014; Laporte et al., 2014). Recent research has shown gene redundancy in the role of ERNs and NF-YAs in the root nodule symbiosis (Cerri et al., 2012, 2016; Laloum et al., 2014; Laporte et al., 2014). Our research has shown that the ipd3l ipd3-2 double mutant exhibits a very severe symbiotic defect phenotype, being unable to form infection threads and nodules (Figure 1) and IPD3L can functionally replace IPD3 when expressed under the control of the IPD3 promoter (Figure 3 ). Thus, we conclude that $I P D 3 L$ is a potential transcription factor that functions redundantly with IPD3 to regulate root nodule symbiosis. These findings also indicate that IPD3 and IPD $3 L$ may possess equivalent biological activities when expressed at similar levels in identical tissues. However, the expression levels and/or tissue specificity of endogenous IPD3L are not sufficient and/or appropriate to functionally replace IPD3, when the IPD3 gene is inactivated (Figures 1, 2). We conclude that the functional specialization of these symbiotic transcription factors might occur primarily via the evolution of promoter specificity rather than by the divergence of protein functions.

In this study, we identified phosphorylation sites (S14, S43, S50, T59, S81, S88, S155, and S407) on IPD3 and found that the IPD3-2D (S50, S155) could rescue the nodule formation on the ipd3l ipd3-2 double mutant roots (Table S3). Strikingly, IPD3$8 \mathrm{D}$, the phosphomimetic sites decreased the nodule number compared to IPD3-WT or IPD3-2D (Tables S3, S4) and the expression level of root nodule symbiotic genes was also decreased in the IPD3-8D transgenic hairy roots. We speculate that the multisite phosphorylation of IPD3 (IPD3-8D) might function as a code to positively or negatively regulate root nodule symbiosis.

Autoactive gain of function variants of CCaMK/DMI3 resulted in spontaneous nodule organogenesis in the absence of rhizobia (Gleason et al., 2006; Tirichine et al., 2006). CYCLOPS$2 \mathrm{D}$ can also trigger the spontaneous formation of root nodules in the absence of rhizobia (Singh et al., 2014). We revealed that IPD3-2D can promote spontaneous nodule formation on ipd3l ipd3-2 hairy roots and also promote spontaneous rootlike nodules on the $d m i 2-1$ and $d m i 3-1$ hairy roots (Figure 5 and Table S5). Furthermore, the spontaneous root-like nodules were not observed on the dmi3-1 roots transformed with the autoactive DMI3 1-311 variant. Our current data indicates that an unknown protein and other phosphorylation sites of IPD3 activated by CCaMK/DMI3 are required to maintain nodule morphogenesis in the root nodule symbiosis (Figure 7).

Previous studies has shown that the ipd3-2 roots showed the presence of intraradical and extraradical hyphae and pigmented

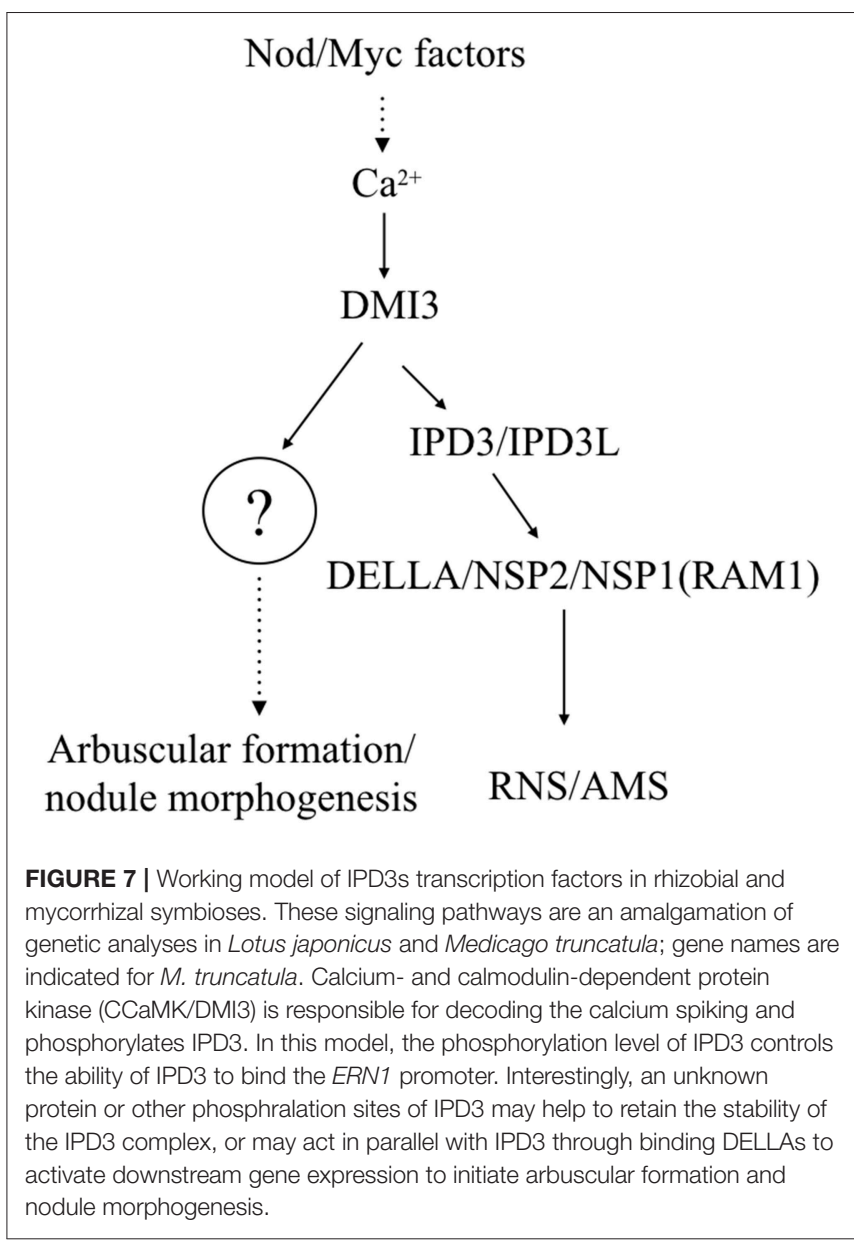

cells, but no arbuscules inoculated with purified Glomus intraradices spores, while The ipd3-1 plants displayed normal arbuscule development with lower frequency of colonization (Yano et al., 2008; Horváth et al., 2011; Pimprikar et al., 2016). Under our condition, fully developed arbuscules were also found in the ipd3-2 and ipd3l ipd3-2 roots inoculated with sands mixed with $R$. intraradices (Figure 6). This different phenotype of ipd32 may be caused by the different AM fungi or the different growth conditions. We also propose that another genetic component, in addition to IPD3/IPD3L, functions downstream of DMI3 to regulate arbuscule development during mycorrhizal symbiosis.

\section{AUTHOR CONTRIBUTIONS}

YJ, EW, and NY conceived the original screening and research plans; YJ performed most of the experiments; YJ, EW, and NY conceived the project and wrote the article with contributions from all the authors.

\section{FUNDING}

This project was supported by National Key R\&D Program of China (2016YFA0500502 to JY), and the National Science 
Foundation for Young Scientists of China (31700208 to YJ, 31500233 to NY).

\section{ACKNOWLEDGMENTS}

We thank Ann Seward, ELS, from Liwen Bianji, Edanz Editing China (www.liwenbianji.cn/ac), for editing the English text of a draft of this manuscript. Thanks

\section{REFERENCES}

Arrighi, J. F., Barre, A., Ben Amor, B., Bersoult, A., Soriano, L. C., Mirabella, R., et al. (2006). The Medicago truncatula lysin motif-receptor-like kinase gene family includes NFP and new nodule-expressed genes. Plant Physiol. 142, 265-279. doi: 10.1104/pp.106.084657

Boisson-Dernier, A., Andriankaja, A., Chabaud, M., Niebel, A., Journet, E. P., Barker, D. G., et al. (2005). MtENOD11 gene activation during rhizobial infection and mycorrhizal arbuscule development requires a common AT-richcontaining regulatory sequence. Mol. Plant Microbe Interact. 18, 1269-1276. doi: 10.1094/MPMI-18-1269

Boisson-Dernier, A., Chabaud, M., Garcia, F., Becard, G., Rosenberg, C., and Barker, D. G. (2001). Agrobacterium rhizogenes-transformed roots of Medicago truncatula for the study of nitrogen-fixing and endomycorrhizal symbiotic associations. Mol. Plant Microbe Interact. 14, 695-700. doi: 10.1094/MPMI.2001.14.6.695

Cerri, M. R., Frances, L., Kelner, A., Fournier, J., Middleton, P. H., Auriac, M. C., et al. (2016). The Symbiosis-related ERN transcription factors act in concert to coordinate rhizobial host root infection. Plant Physiol. 171, 1037-1054. doi: 10.1104/pp.16.00230

Cerri, M. R., Frances, L., Laloum, T., Auriac, M. C., Niebel, A., Oldroyd, G. E., et al. (2012). Medicago truncatula ERN transcription factors: regulatory interplay with NSP1/NSP2 GRAS factors and expression dynamics throughout rhizobial infection. Plant Physiol. 160, 2155-2172. doi: 10.1104/pp.112.2 03190

Chen, C., Ane, J. M., and Zhu, H. (2008). OsIPD3, an ortholog of the Medicago truncatula DMI3 interacting protein IPD3, is required for mycorrhizal symbiosis in rice. New Phytol. 180, 311-315. doi: 10.1111/j.1469-8137.2008.02612.x

Couzigou, J. M., Zhukov, V., Mondy, S., Abuel Heba, G., Cosson, V., Ellis, T. H., et al. (2012). NODULE ROOT and COCHLEATA maintain nodule development and are legume orthologs of Arabidopsis BLADE-ON-PETIOLE genes. Plant Cell 24, 4498-4510. doi: 10.1105/tpc.112.103747

de Lucas, M., Daviere, J. M., Rodriguez-Falcon, M., Pontin, M., Iglesias-Pedraz, J. M., Lorrain, S., et al. (2008). A molecular framework for light and gibberellin control of cell elongation. Nature 451, 480-484. doi: 10.1038/nature06520

Feng, S., Martinez, C., Gusmaroli, G., Wang, Y., Zhou, J., Wang, F., et al. (2008). Coordinated regulation of Arabidopsis thaliana development by light and gibberellins. Nature 451, 475-479. doi: 10.1038/nature06448

Fonouni-Farde, C., Tan, S., Baudin, M., Brault, M., Wen, J., Mysore, K. S., et al. (2016). DELLA- mediated gibberellin signalling regulates Nod factor signalling and rhizobial infection. Nat. Commun. 7:12636. doi: 10.1038/ncomms12636

Gleason, C., Chaudhuri, S., Yang, T., Munoz, A., Poovaiah, B. W., and Oldroyd, G. E. (2006). Nodulation independent of rhizobia induced by a calcium-activated kinase lacking autoinhibition. Nature 441, 1149-1152. doi: $10.1038 /$ nature 04812

Goodstein, D. M., Shu, S., Howson, R., Neupane, R., Hayes, R. D., Fazo, J., et al. (2012). Phytozome: a comparative platform for green plant genomics. Nucleic Acids Res. 40, D1178-D1186. doi: 10.1093/nar/gkr944

Grimsrud, P. A., den Os, D., Wenger, C. D., Swaney, D. L., Schwartz, D., Sussman, M. R., et al. (2010). Large-scale phosphoprotein analysis in Medicago truncatula roots provides insight into in vivo kinase activity in legumes. Plant Physiol. 152, 19-28. doi: $10.1104 / p p .109 .149625$

Horváth, B., Yeun, L. H., Domonkos, A., Halasz, G., Gobbato, E., Ayaydin, F., et al. (2011). Medicago truncatula IPD3 is a member of the common symbiotic to members in Wang's lab involved in the helpful discussion.

\section{SUPPLEMENTARY MATERIAL}

The Supplementary Material for this article can be found online at: https://www.frontiersin.org/articles/10.3389/fpls.2018. 00267/full\#supplementary-material signaling pathway required for rhizobial and mycorrhizal symbioses. Mol. Plant Microbe Interact. 24, 1345-1358. doi: 10.1094/MPMI-01-11-0015

Javot, H., Penmetsa, R. V., Terzaghi, N., Cook, D. R., and Harrison, M. J. (2007). A Medicago truncatula phosphate transporter indispensable for the arbuscular mycorrhizal symbiosis. Proc. Natl. Acad. Sci. U.S.A. 104, 1720-1725. doi: 10.1073/pnas.0608136104

Jiang, Y., Wang, W., Xie, Q., Liu, N., Liu, L., Wang, D., et al. (2017). Plants transfer lipids to sustain colonization by mutualistic mycorrhizal and parasitic fungi. Science 356, 1172-1175. doi: 10.1126/science.aam9970

Jin, Y., Liu, H., Luo, D., Yu, N., Dong, W., Wang, C., et al. (2016). DELLA proteins are common components of symbiotic rhizobial and mycorrhizal signalling pathways. Nat. Commun. 7:12433. doi: 10.1038/ncomms12433

Kalo, P., Gleason, C., Edwards, A., Marsh, J., Mitra, R. M., Hirsch, S., et al. (2005). Nodulation signaling in legumes requires NSP2, a member of the GRAS family of transcriptional regulators. Science 308, 1786-1789. doi: $10.1126 /$ science. 1110951

Kistner, C., Winzer, T., Pitzschke, A., Mulder, L., Sato, S., Kaneko, T., et al. (2005). Seven Lotus japonicus genes required for transcriptional reprogramming of the root during fungal and bacterial symbiosis. Plant Cell 17, 2217-2229. doi: $10.1105 /$ tpc. 105.032714

Laloum, T., Baudin, M., Frances, L., Lepage, A., Billault-Penneteau, B., Cerri, M. R., et al. (2014). Two CCAAT-box-binding transcription factors redundantly regulate early steps of the legume-rhizobia endosymbiosis. Plant J. 79, 757-768. doi: $10.1111 /$ tpj.12587

Laporte, P., Lepage, A., Fournier, J., Catrice, O., Moreau, S., Jardinaud, M. F., et al. (2014). The CCAAT box-binding transcription factor NF-YA1 controls rhizobial infection. J. Exp. Bot. 65, 481-494. doi: 10.1093/jxb/ert392

Levy, J., Bres, C., Geurts, R., Chalhoub, B., Kulikova, O., Duc, G., et al. (2004). A putative $\mathrm{Ca}^{2+}$ and calmodulin-dependent protein kinase required for bacterial and fungal symbioses. Science 303, 1361-1364. doi: 10.1126/science.1093038

Limpens, E., Franken, C., Smit, P., Willemse, J., Bisseling, T., and Geurts, R. (2003). LysM domain receptor kinases regulating rhizobial Nod factor-induced infection. Science 302, 630-633. doi: 10.1126/science.1090074

Luginbuehl, L. H., Menard, G. N., Kurup, S., Van Erp, H., Radhakrishnan, G. V., Breakspear, A., et al. (2017). Fatty acids in arbuscular mycorrhizal fungi are synthesized by the host plant. Science 356, 1175-1178. doi: $10.1126 /$ science.aan0081

Madsen, E. B., Madsen, L. H., Radutoiu, S., Olbryt, M., Rakwalska, M., Szczyglowski, K., et al. (2003). A receptor kinase gene of the LysM type is involved in legume perception of rhizobial signals. Nature 425, 637-640. doi: 10.1038 /nature 02045

McGonigle, T. P., Miller, M. H., Evans, D. G., Fairchild, G. L., and Swan, J. A. (1990). A new method that gives an objective measure of colonization of roots by vesicular-arbuscular mycorrhizal fungi. New Phytol. 115, 495-501. doi: 10.1111/j.1469-8137.1990.tb00476.x

Messinese, E., Mun, J. H., Yeun, L. H., Jayaraman, D., Rouge, P., Barre, A., et al. (2007). A novel nuclear protein interacts with the symbiotic DMI3 calciumand calmodulin-dependent protein kinase of Medicago truncatula. Mol. Plant Microbe Interact. 20, 912-921. doi: 10.1094/MPMI-20-8-0912

Middleton, P. H., Jakab, J., Penmetsa, R. V., Starker, C. G., Doll, J., Kalo, P., et al. (2007). An $E R F$ transcription factor in Medicago truncatula that is essential for Nod factor signal transduction. Plant Cell 19, 1221-1234. doi: $10.1105 /$ tpc. 106.048264

Mitra, R. M., Gleason, C. A., Edwards, A., Hadfield, J., Downie, J. A., Oldroyd, G. E., et al. (2004). A Ca ${ }^{2+} /$ calmodulin-dependent protein kinase required for 
symbiotic nodule development: gene identification by transcript-based cloning. Proc. Natl. Acad. Sci. U.S.A. 101, 4701-4705. doi: 10.1073/pnas.0400595101

Oldroyd, G. E. (2013). Speak, friend, and enter: signalling systems that promote beneficial symbiotic associations in plants. Nat. Rev. Microbiol. 11, 252-263. doi: 10.1038/nrmicro2990

Ovchinnikova, E., Journet, E. P., Chabaud, M., Cosson, V., Ratet, P., Duc, G., et al. (2011). IPD3 controls the formation of nitrogen-fixing symbiosomes in pea and Medicago Spp. Mol. Plant Microbe Interact. 24, 1333-1344. doi: 10.1094/MPMI-01-11-0013

Parniske, M. (2008). Arbuscular mycorrhiza: the mother of plant root endosymbioses. Nat. Rev. Microbiol. 6, 763-775. doi: 10.1038/nrmicro1987

Pimprikar, P., Carbonnel, S., Paries, M., Katzer, K., Klingl, V., Bohmer, M. J., et al. (2016). A CCaMK-CYCLOPS-DELLA complex activates transcription of RAM1 to regulate arbuscule branching. Curr. Biol. 26, 987-998. doi: 10.1016/j.cub.2016.04.021

Radutoiu, S., Madsen, L. H., Madsen, E. B., Felle, H. H., Umehara, Y., Gronlund, M., et al. (2003). Plant recognition of symbiotic bacteria requires two LysM receptor-like kinases. Nature 425, 585-592. doi: 10.1038/nature02039

Singh, S., Katzer, K., Lambert, J., Cerri, M., and Parniske, M. (2014). CYCLOPS, a DNA-binding transcriptional activator, orchestrates symbiotic root nodule development. Cell Host Microbe 15, 139-152. doi: 10.1016/j.chom.2014. 01.011

Smit, P., Raedts, J., Portyanko, V., Debelle, F., Gough, C., Bisseling, T., et al. (2005). NSP1 of the GRAS protein family is essential for rhizobial Nod factor-induced transcription. Science 308, 1789-1791. doi: 10.1126/science. 1111025

Soyano, T., Hirakawa, H., Sato, S., Hayashi, M., and Kawaguchi, M. (2014). Nodule inception creates a long-distance negative feedback loop involved in homeostatic regulation of nodule organ production. Proc. Natl. Acad. Sci. U.S.A. 111, 14607-14612. doi: 10.1073/pnas.1412716111

Tamura, K., Peterson, D., Peterson, N., Stecher, G., Nei, M., and Kumar, S. (2011). MEGA5: molecular evolutionary genetics analysis using maximum likelihood, evolutionary distance, and maximum parsimony methods. Mol. Biol. Evol. 28, 2731-2739. doi: 10.1093/molbev/ msr121
Tirichine, L., Imaizumi-Anraku, H., Yoshida, S., Murakami, Y., Madsen, L. H., Miwa, H., et al. (2006). Deregulation of a $\mathrm{Ca}^{2+} /$ calmodulin-dependent kinase leads to spontaneous nodule development. Nature 441, 1153-1156. doi: $10.1038 /$ nature 04862

Wang, E., Yu, N., Bano, S. A., Liu, C., Miller, A. J., Cousins, D., et al. (2014). $\mathrm{A} \mathrm{H}^{+}$-ATPase that energizes nutrient uptake during mycorrhizal symbioses in rice and Medicago truncatula. Plant Cell 26, 1818-1830. doi: 10.1105/tpc.113.120527

Wang, W., Shi, J., Xie, Q., Jiang, Y., Yu, N., and Wang, E. (2017). Nutrient exchange and regulation in arbuscular mycorrhizal symbiosis. Mol. Plant 10, 1147-1158. doi: 10.1016/j.molp.2017.07.012

Yano, K., Yoshida, S., Muller, J., Singh, S., Banba, M., Vickers, K., et al. (2008). CYCLOPS, a mediator of symbiotic intracellular accommodation. Proc. Natl. Acad. Sci. U.S.A. 105, 20540-20545. doi: 10.1073/pnas.0806858105

Yoo, S. D., Cho, Y. H., and Sheen, J. (2007). Arabidopsis mesophyll protoplasts: a versatile cell system for transient gene expression analysis. Nat. Protoc. 2, 1565-1572. doi: 10.1038/nprot.2007.199

Zhang, X. C., Wu, X., Findley, S., Wan, J., Libault, M., Nguyen, H. T., et al. (2007). Molecular evolution of lysin motif-type receptor-like kinases in plants. Plant Physiol. 144, 623-636. doi: 10.1104/pp.107.097097

Zhang, X., Dong, W., Sun, J., Feng, F., Deng, Y., He, Z., et al. (2015). The receptor kinase CERK1 has dual functions in symbiosis and immunity signalling. Plant J. 81, 258-267. doi: $10.1111 /$ tpj.12723

Conflict of Interest Statement: The authors declare that the research was conducted in the absence of any commercial or financial relationships that could be construed as a potential conflict of interest.

Copyright $\odot 2018$ Jin, Chen, Yang, Mysore, Wen, Huang, Yu and Wang. This is an open-access article distributed under the terms of the Creative Commons Attribution License (CC BY). The use, distribution or reproduction in other forums is permitted, provided the original author(s) and the copyright owner are credited and that the original publication in this journal is cited, in accordance with accepted academic practice. No use, distribution or reproduction is permitted which does not comply with these terms. 